\title{
ON A GENERALIZATION OF U-MEANS
}

\author{
FRANCOIS DUBEAU \\ Département de mathématiques \\ Collège militaire royal de Saint-Jean \\ Saint-Jean-sur-Richelieu, Québec, Canada, JOJ 1RO
}

(Received March 14, 1990, and in revised form October 2, 1990)

\begin{abstract}
In this paper we present an extension of Bauer's work about u-means. We consider a kind of composition of an admissible function $u(x)$ (described by Bauer) and of a compatible function $\phi(x)$. This construction allows us to define $(u, \phi)$-means. When $\phi(x)=x$, the $(u, \phi)$-means are the $u$-means introduced by Bauer. The arithmetic, geometric and harmonic means are special cases.
\end{abstract}

KEY WORDS AND PHRASES. Means, u-means, generalized u-means. 1980 AMS SUBJECT CLASSIFICATION CODES. 26D99, 39B99.

\section{INTRODUCTION.}

In [2] Bauer introduced a class of admissible functions. To each function $u(x)$ in this class it was possible to associate a $u$-mean. The arithmetic and geometric means were special cases of $u$ means but not the harmonic mean.

In this paper we introduce a class of monotone compatible functions. We consider a kind of composition of an admissible function $u(x)$ and a monotone function $\phi(x)$ compatible with respect to $u(x)$ which permits the definition of $(u, \phi)$-means. When $\phi(x)=x$ the $(u, \phi)$-means are the $u$ means of Bauer. The arithmetic, geometric and harmonic means are special cases.

2. CONTRACTIVE INTERVAL.

In this paper we consider intervals $I \subset[0,+\infty[$ of the following type

(i) $\quad] 0,+\infty[$,

(ii) $\quad] 0, \alpha]$ or $] 0, \alpha[\quad$ for $0<\alpha \leq 1$,

(iii) $\quad[\beta,+\infty[$ or $] \beta,+\infty[$ for $1 \leq \beta<+\infty$.

Any interval $I$ of this type is said to be contractive because for any $n \in N=\{1,2,3, \cdots\}$ and $x \in I$ we have $x^{n} \in I$, or equivalently, for any $x, y \in I$ we have $x y \in I$ (see [2]).

3. CLASSES OF FUNCTIONS. $[2]$.

The first class of functions we consider is the class of admissible functions introduced by Bauer

A strictly positive continuous function $u(x)$ defined on a contractive interval $I_{u}$ is said admissible (of type (A) or (B)) if it satisfies one of the following conditions:

(A) $x \rightarrow u(x)$ is decreasing,

(B) $\quad x \rightarrow u(x) / x$ is strictly increasing.

EXAMPLE 1. $u(x)=x^{p}$ for $p \leq 0$ or $p>1$ are admissible functions on $I_{u}$. The function $u(x)=\sqrt{1-x^{2}}$ is admissible on $\left.I_{u}=\right] 0,1\left[\right.$. The function $u(x)=e^{x-1}$ is admissible on $[1,+\infty[$.

To extend the work of Bauer we introduce the following class of functions. A strictly positive 
strictly monotone continuous function $\phi(x)$ defined on a contractive interval $I_{\phi}$ is said compatible if it satisfies the following condition:

$$
x \rightarrow \phi(\alpha x) / \phi(x) \text { is monotone (as } \phi(x)) \text { for any } \alpha \in I_{\phi} .
$$

Let us consider the following examples.

EXAMPLE 2. $\phi(x)=x^{p}$ is strictly increasing for $p>0$ and strictly decreasing for $p<0$. Also $\phi(\alpha x) / \phi(x)=\alpha^{p}$, a constant for any fixed $\alpha \in I_{\phi}$. Note that in this case $\phi\left(x^{n}\right)=[\phi(x)]^{n}$.

EXAMPLE 3. $\phi(x)=e^{x}$ and $I_{\phi}=[1,+\infty[$. The function $\phi(x)$ is a strictly increasing continuous function such that $\phi(\alpha x) / \phi(x)=e^{(\alpha-1) x}$ which is an increasing function for any fixed $\alpha \in I_{\phi}$.

EXAMPLE 4. $\phi(x)=e^{-\frac{1}{x}}$ and $\left.\left.I_{\phi}=\right] 0,1\right]$. The function $\phi(x)$ is a strictly increasing function on $I_{\phi}$ such that $\phi(\alpha x) / \phi(x)=e^{-\left(\frac{1-\alpha}{\alpha x}\right)}$ which is an increasing function for any fixed $\alpha \in I_{\phi}$.

The following preliminary results will be useful in the next section.

LEMMA 1. Let $\phi(x)$ be a compatible function. Then

(i) $x \rightarrow \phi\left(x^{n}\right) / \phi(x)$ is strictly monotone (as $\left.\phi(x)\right)$ for any integer $n \in N$,

(ii) $x \rightarrow \phi\left(\alpha^{n} x\right) / \phi(x)$ is monotone (as $\left.\phi(x)\right)$ for any $n \in N$ and any fixed $\alpha \in I_{\phi}$.

PROOF. Let us assume first that $\phi(x)$ is strictly increasing. To prove (i) consider $x<y$, then $x^{n}<x^{n-1} y<x y^{n-1}<y^{n}$. Hence $\phi\left(x^{n}\right) / \phi(x)<\phi\left(x y^{n-1}\right) / \phi(x) \leq \phi\left(y^{n}\right) / \phi(y)$ because $\phi(x)$ is strictly increasing and compatible. To prove (ii) replace $\alpha$ by $\alpha^{n}$ in the definition. The proof is almost the same when $\phi(x)$ is strictly decreasing.

LEMMA 2. Let $u(x)$ be an admissible function and $\phi(x)$ be a compatible function. If $\phi(I(\phi)) \subset I_{u}$, then for any $n \in N$ the function $x \rightarrow \psi_{n+1}(x)=u o \phi\left(x^{n}\right) / \phi(x)$ is strictly monotone (here $u o \phi(x)=u(\phi(x)))$. The different cases are summarized in the following table:

type of $u(x)$ $\phi(x)$ strictly monotone

increasing

decreasing

increasing

decreasing $\psi_{n+1}(x)$ strictly monotone

decreasing

increasing

increasing decreasing

PROOF. Let us assume that $\phi(x)$ is strictly increasing (decreasing). If $u(x)$ is of type (A) then $u o \phi(x)$ is decreasing (increasing). Also $1 / \phi(x)$ is strictly decreasing (increasing). It follows that $u o \phi\left(x^{n}\right) / \phi(x)$ is strictly decreasing (increasing). If $u(x)$ is of type (B) then $u o \phi(x) / \phi(x)$ is strictly increasing (decreasing). From Lemma $1, \phi\left(x^{n}\right) / \phi(x)$ is strictly increasing (decreasing). The result follows from $u o \phi\left(x^{n}\right) / \phi(x)=\left[\operatorname{uo\phi }\left(x^{n}\right) / \phi\left(x^{n}\right)\right]\left[\phi\left(x^{n}\right) / \phi(x)\right]$

4. $(u, \phi)$-MEANS. Let $u(x)$ be an admissible function and $\phi(x)$ be a compatible function such that $\phi\left(I_{\phi}\right) \subset I_{u}$. Let $n \geq 2$ and choose any $\vec{a}=\left(a_{1}, a_{2}, \cdots, a_{n}\right) \in I_{\phi}^{n}=I_{\phi} \times \cdots \times I_{\phi}$. We consider

where

$$
S_{(u, \phi)}(\vec{a})=\frac{\sum_{i=1}^{n} \operatorname{uo\phi }\left(\pi_{i}(\vec{a})\right)}{\sum_{i=1}^{n} \phi\left(a_{i}\right)}
$$

$$
\pi_{i}(\vec{a})=\prod_{\substack{j=1 \\ j \neq i}}^{n} a_{j}=\left(\prod_{j=1}^{n} a_{j}\right) / a_{i} .
$$

Using now the continuity of the functions and the strict monotonicity of $\psi_{n}(x)=u o \phi\left(x^{n-1}\right) / \phi(x)$, we can prove the following result (which is a generalization of Theorem 2.1 of Bauer). 
THEOREM 3. Let $u(x)$ be an admissible function defined on $I_{u}$ and $\phi(x)$ a compatible function defined on $I_{\phi}$ such that $\phi\left(I_{\phi}\right) \subset I_{u}$. Let $n \geq 2$ and $\vec{a}=\left(a_{1}, \cdots, a_{n}\right) \in I_{\phi}^{n}$. Then the equation

$$
\psi_{n}(x)=S_{(u, \phi)}(\vec{a})
$$

has exactly one solution in $I_{\phi}$. It lies in the interval

$$
] \alpha, \beta\left[\text { if } \alpha=\min \left\{a_{1}, \cdots, a_{n}\right\}<\max \left\{a_{1}, \cdots, a_{n}\right\}=\beta\right.
$$

and is equal to $\alpha$ if $\alpha=\beta$.

NOTE. With the assumptions made on $\phi(x)$ and the preceding two lemmas, the proof of this theorem is almost identical to the proof of Theorem 2.1 of Bauer.

PROOF. If $\alpha=\beta$ the result follows from Lemma 2. Let us assume that $\alpha=\beta$ and let us consider the following two cases:

(i) $u(x)$ is of type $(\mathrm{A})$ and $\phi(x)$ is strictly decreasing. In this case uo $\phi(x)$ is increasing. For any $i=1, \cdots, n$ we have $\alpha^{n-1} \leq \pi_{i}(\vec{a}) \leq \beta^{n-1}$ and it follows that $u o \phi\left(\alpha^{n-1}\right) \leq u o \phi\left(\pi_{i}(\vec{a})\right) \leq u o \phi\left(\beta^{n-1}\right)$. Also $1 / \phi(x)$ is strictly increasing, then we have $\phi\left(a_{i}\right) / \phi(\alpha) \leq 1 \leq \phi\left(a_{i}\right) / \phi(\beta)$ with strict inequality for at least one $i$ (not necessarily the same $i$ for both inequalities). It follows that

$$
\phi\left(a_{i}\right) \psi_{n}(\alpha) \leq u o \phi\left(\pi_{i}(\vec{a})\right) \leq \phi\left(a_{i}\right) \psi_{n}(\beta)
$$

for $i=1, \cdots, n$.

(ii) $u(x)$ is of type (B) and $\phi(x)$ is structly increasing. In this case $u o \phi(x) / \phi(x)$ is strictly increasing and we have

$$
\frac{u \circ \phi\left(\alpha^{n-1}\right)}{\phi\left(\alpha^{n-1}\right)} \leq \frac{u o \phi\left(\pi_{i}(\vec{a})\right)}{\phi\left(\pi_{i}(\vec{a})\right)} \leq \frac{u o \phi\left(\beta^{n-1}\right)}{\phi\left(\beta^{n-1}\right)}
$$

and again with strict inequality for at least one $i$. We also have

$$
\phi\left(\alpha^{n-2} a_{i+1}\right) \leq \phi\left(\pi_{i}(\vec{a})\right) \leq \phi\left(\beta^{n-2} a_{i+1}\right)
$$

for $i=1, \cdots, n$ (where $\left.a_{n+1} \equiv a_{1}\right)$. From Lemma 1 we have

$$
\phi\left(\alpha^{n-1}\right) / \phi(\alpha) \leq \phi\left(\alpha^{n-2} a_{i+1}\right) / \phi\left(a_{i+1}\right) \text { and } \phi\left(\beta^{n-2} a_{i+1}\right) / \phi\left(a_{i+1}\right) \leq \phi\left(\beta^{n-1}\right) / \phi(\beta) .
$$

It follows that

for $i=1, \cdots, n$.

$$
\phi\left(a_{i+1}\right) \psi_{n}(\alpha) \leq u o \phi\left(\pi_{i}(\vec{a})\right) \leq \phi\left(a_{i+1}\right) \psi_{n}(\beta)
$$

By adding up (4.2) or (4.3) for $i=1, \cdots, n$ it follows that $\psi_{n}(\alpha)<S_{(u, \phi)}(\vec{a})<\psi_{n}(\beta)$ and the result follows from the continuity and the strict monotonicity of $\psi_{n}(x)$.

For the other cases we obtain reverse inequalities and the result follows again.

Under the assumptions of Theorem 3, the $(u, \phi)$-mean of the $n$ numbers $a_{1}, \cdots, a_{n}$ taken in $I_{\phi}$ will be the unique solution of (4.1) and will be denoted $M_{(u, \phi)}(\vec{a})$. If $n=1$ we put $M_{(u, \phi)}\left(a_{1}\right)=a_{1}$.

REMARK 1 . The u-means introduced by Bauer, denoted $M_{u}(\vec{a})$, are obtained when $\phi(x)$ is the identity function $\mathrm{id}(x)$, i.e. $\phi(x)=\mathrm{id}(x)=x$ for any $x \in I_{\phi}$, and we have $M_{(u, i d)}(\vec{a})=M_{u}(\vec{a})$.

REMARK 2. For $u(x)=1$ we have 


$$
\phi\left(M_{(1, \phi)}(\vec{a})\right)=A(\phi(\vec{a}))
$$

where $\phi(\vec{a})$ denotes the vector $\left(\phi\left(a_{1}\right), \cdots, \phi\left(a_{n}\right)\right)$ and $A(\vec{v})$ is the arithmetic mean of the $n$ components of the vector $\vec{v}=\left(v_{1}, \cdots, v_{n}\right)$.

EXAMPLE 5. Consider $u(x)=1$. For $\phi(x)=\mathrm{id}(x)=x$ we obtain

$$
M_{(1, \mathrm{id})}(\vec{a})=A(\vec{a})
$$

and for $\phi(x)=1 / x=(1 /$ id $(x)$ since

$$
H\left(a_{1}, \cdots, a_{n}\right)^{-1}=A\left(a_{1}^{-1}, \cdots, a_{n}^{-1}\right)
$$

it follows from (4.4) that

$$
M_{(1,1 / \mathrm{id})}(\vec{a})=H(\vec{a})
$$

where $H(\vec{a})$ denotes the harmonic mean of the $n$ components of $\vec{a}$. Let us note that it is not possible to obtain the harmonic mean as a u-means (see [1], [2]).

REMARK 3. More generally, if the function $\phi(x)$ is such that

then we have

$$
\phi\left(\prod_{i=1}^{n} a_{i}\right)=\prod_{i=1}^{n} \phi\left(a_{i}\right)
$$

and it follows that

$$
S_{(u, \phi)}(\vec{a})=S_{(u, \mathrm{id})}(\phi(\vec{a}))
$$

$$
\phi\left(M_{(u, \phi)}(\vec{a})\right)=M_{(u, \mathrm{id})}(\phi(a))=M_{u}(\phi(\vec{a})) .
$$

EXAMPLE 6. For $u(x)=1 / x=(1 / \mathrm{id})(x)$, if $\phi(x)=\mathrm{id}(x)$ we obtain

$$
M_{(1 / \mathrm{id}, \mathrm{id})}(\vec{a})=G(\vec{a})
$$

where $G(\vec{a})$ is the geometric mean of the $n$ components of $\vec{a}$. If $\phi(x)=1 / x=(1 / \mathrm{id})(x)$ it follows from (4.5) that

$$
M_{(1 / \mathrm{id}, 1 / \mathrm{id})}(\vec{a})=G(\vec{a})
$$

because $G\left(a_{1}^{-1}, \cdots, a_{n}^{-1}\right)=G\left(a_{1}, \cdots, a_{n}\right)^{-1}$.

EXAMPLE 7. More generally if $u_{p}(x)=x^{p}$ (for $p \leq 0$ or $p>1$ ) and $\phi(x)=x=\operatorname{id}(x)$ we have

$$
M_{\left(u_{p}, \text { id }\right)}(\vec{a})=\left[\frac{G^{n p}\left(a_{1}, \cdots, a_{n}\right)}{A\left(a_{1}, \cdots, a_{n}\right) H\left(a_{1}, \cdots, a_{n}^{p}\right)}\right]^{\frac{1}{p n-p-1}}
$$

(see [2]). For $\phi(x)=1 / x=(1 /$ id $)(x)$ we have

$$
\begin{aligned}
M_{\left(u_{p}, 1 / \mathrm{id}\right)}(\vec{a}) & \left.=M_{\left(u_{p}, \mathrm{id}\right)}\left(\frac{1}{\mathrm{id}}(\vec{a})\right)\right)^{-1} \\
& =\left[\frac{G^{n p}\left(a_{1}, \cdots, a_{n}\right)}{A\left(a_{1}^{p}, \cdots, a_{n}^{p}\right) H\left(a_{1}, \cdots, a_{n}\right)}\right] \frac{1}{p n-p-1}
\end{aligned}
$$


EXAMPLE 8. Consider $\phi(x)=e^{x}$ on $I_{\phi}=[1,+\infty[$. If $u(x)=1$ then

$$
M_{(1, \phi)}(\vec{a})=\ln \left(\frac{1}{n} \sum_{i=1}^{n} e^{a_{i}}\right)=\ln \left(A\left(\phi\left(a_{1}\right), \cdots, \phi\left(a_{n}\right)\right)\right) .
$$

More generally, if $u_{p}(x)=x^{p}(p \leq 0$ or $>1)$ we have that $M_{\left(u_{p}, \phi\right)}(\vec{a})$ is the unique positive solution,
not smaller than 1 , of the polynomial equation

$$
p M^{n-1}-M=\ln \left[\frac{\sum_{i=1}^{\mathrm{n}} \exp \left(\stackrel{\mathrm{n}}{\mathrm{n}}_{1} a_{j} / a_{i}\right)}{\sum_{i=1}^{n} \exp \left(a_{i}\right)}\right]
$$

5. APPLICATIONS TO INEQUALITIES. In [2] Bauer presented inequalities between umeans (or ( $u$, id)-means) and the arithmetic mean. Using the relation (4.5) it is possible to obtain similar inequalities for the harmonic mean. In fact we have the following results.

THEOREM 4. Let $u(x)$ be a convex admissible function of type (A) defined on the interval $I_{u} \supset \phi\left(I_{\phi}\right)$. For every choice of finitely many numbers $a_{1}, \cdots, a_{n} \in I_{\phi}$, if

(i) $\phi(x)=\mathrm{id}(x) \quad$ then $M_{(u, \text { id })}\left(a_{1}, \cdots, a_{n}\right) \leq A\left(a_{1}, \cdots, a_{n}\right)$,

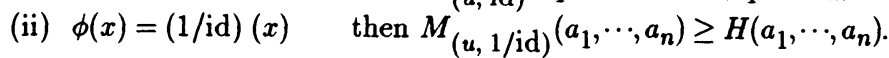

Moreover if $u(x)$ is strictly convex then strict inequalities hold provided that $a_{1}, \cdots, a_{n}$ are not all equal.

THEOREM 5. Let $u(x)$ be a concave admissible function of type (B) defined on $I_{u} \supset \phi\left(I_{\phi}\right)$. For every choice of finitely many numbers $a_{1}, \cdots, a_{n} \in I_{\phi}$, if

(i) $\phi(x)=\operatorname{id}(x) \quad$ then $M_{(u, \text { id })}\left(a_{1}, \cdots, a_{n}\right) \leq A\left(a_{1}, \cdots, a_{n}\right)$,

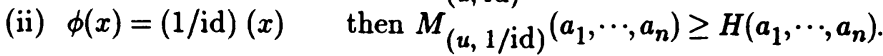

Strict inequalities hold for $n \geq 3$ provided that $a_{1}, \cdots, a_{n}$ are not all equal.

The parts (i) of these two theorems are the results presented by Bauer in [2] because $M_{u}(\vec{a})=M_{(u, \text { id) }}(\vec{a})$. To prove the parts (ii) we only have to consider the relation (4.5) to obtain

$$
M_{(u, 1 / \mathrm{id})}\left(a_{1}, \cdots, a_{n}\right)=1 / M_{u}\left(a_{1}^{-1}, \cdots, a_{n}^{-1}\right) .
$$

Then, using the parts (i) we have

$$
M_{u}\left(a_{1}^{-1}, \cdots, a_{1}^{-1}\right) \leq A\left(a_{1}^{-1}, \cdots, a_{n}^{-1}\right)
$$

but $A\left(a_{1}^{-1}, \cdots, a_{n}^{-1}\right)=H\left(a_{1}, \cdots, a_{n}\right)^{-1}$ and the results follow.

* This work was supported by the Department of National Defence of Canada.

\section{REFERENCES}

1. ACZEL, J., "Related functional equations applied to Korovkin approximation and to the characterization of Renyi entropies - links to the uniqueness theory", C.R. Math. Rep. Acad. Sci. Canada VI (1984), 319-336.

2. BAUER, H., "A class of means and related inequalities", Manuscripta Mathematica 55 (1986), 199-211.

3. HARDY, G.H., LITTLEWOOD, J.E. and POLYA, G., Inequalities, Cambridge Univ. Press, 1934. 


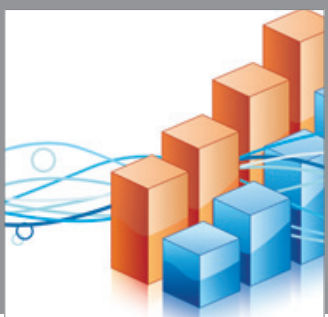

Advances in

Operations Research

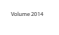

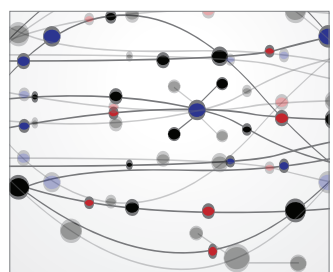

\section{The Scientific} World Journal
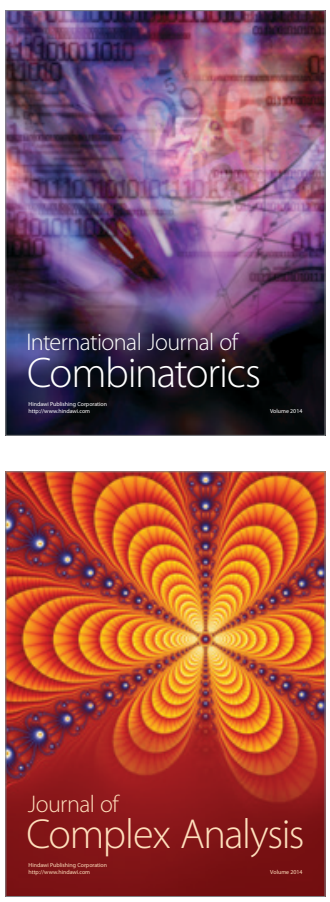

International Journal of

Mathematics and

Mathematical

Sciences
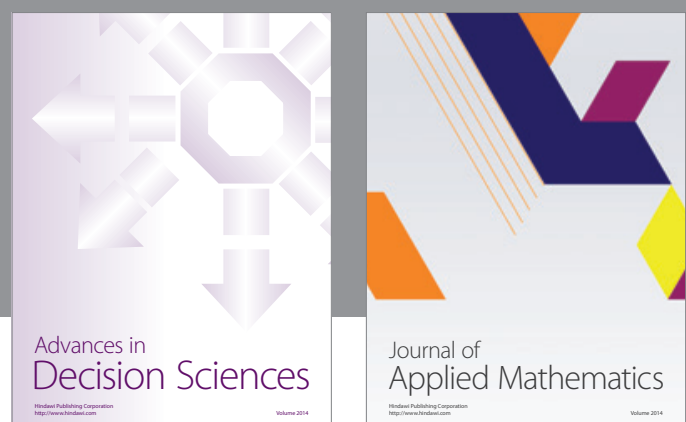

Journal of

Applied Mathematics
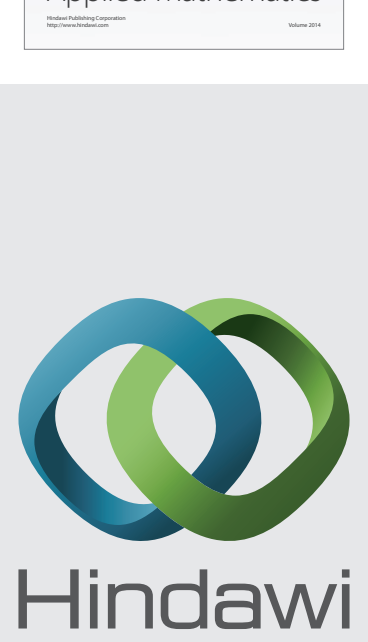

Submit your manuscripts at http://www.hindawi.com
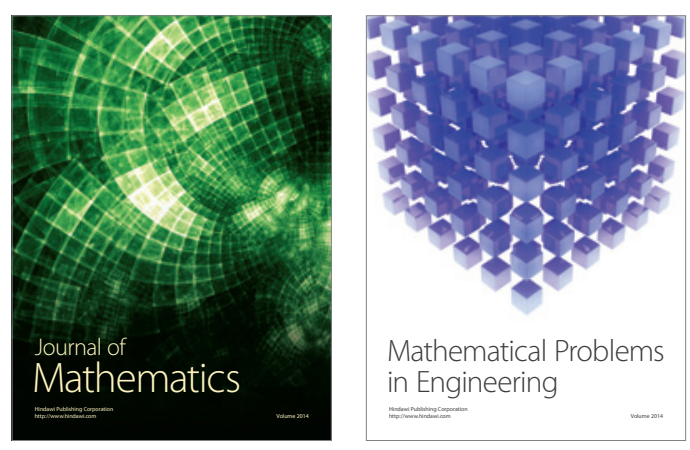

Mathematical Problems in Engineering
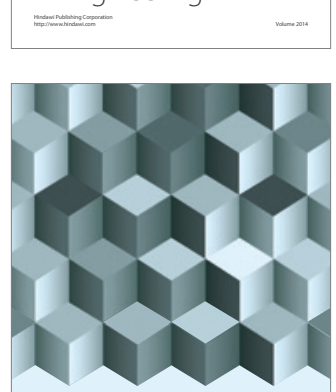

Journal of

Function Spaces
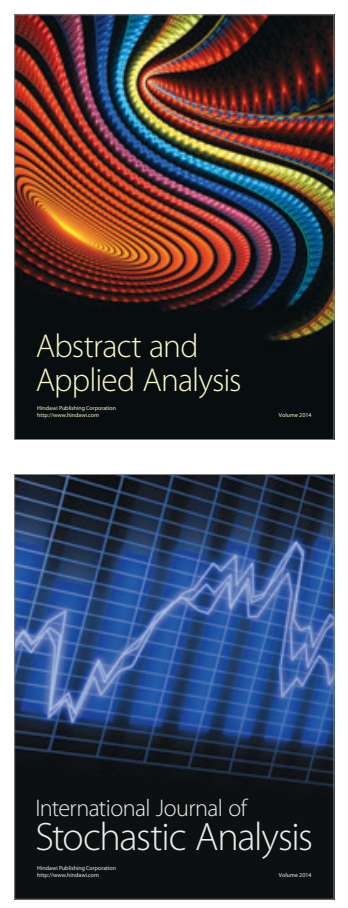

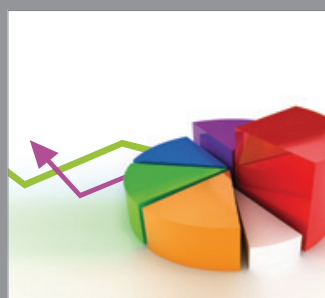

ournal of

Probability and Statistics

Promensencen
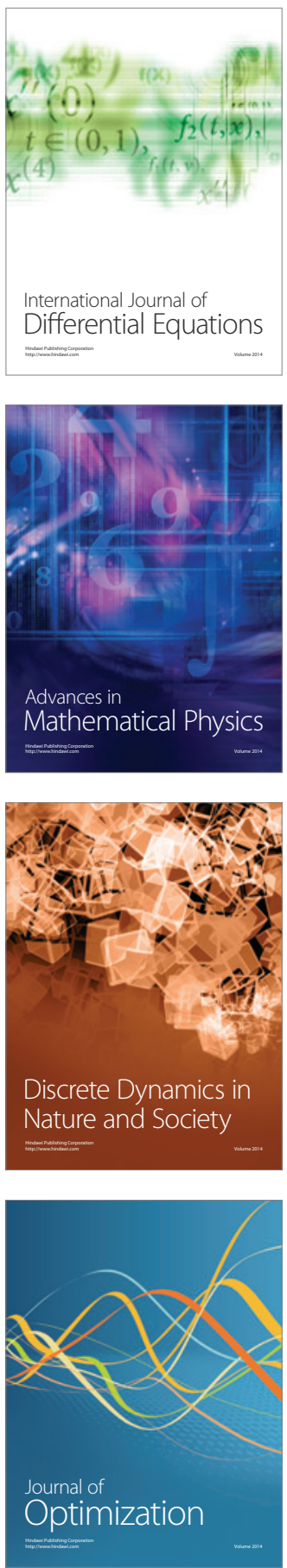